\title{
JULES SUPERVIELLE, LE RÊVEUR ATTENTIF
}

\author{
María Muñoz Romero
}

La sensation d'originalité qui émane de l'oeuvre de Jules Supervielle est incontestable. Le lecteur qui ouvre pour la première fois un recueil de poèmes comme Gravitations ou un livre de contes comme L'Enfant de la Haute Mer se voit d'emblée plongé dans un monde nouveau qui le déconcerte et le surprend. Mais ce qui retient le lecteur et le ramène à Supervielle c'est l'authenticité de cette originalité, sa pureté, sa simplicité non fabriquée. Une originalitée innée. En effet, il ne s'agit nullement d'une recherche à tout prix de l'originalité. Son originalité est dans son imagination.

Toutefois, chez Supervielle originalité ne veut pas dire extravagance ou excentricité. Supervielle n'apporte pas tellement de thèmes inédits, mais bien plutôt une manière nouvelle d'aborder l'univers. Jules Supervielle est le «hors-venu» qui ne cesse de tout voir avec l'émerveillement d'une âme candide. Il ne s'habitue à rien. Il a le don de l'innocence. Pour lui le monde reste établi «sur des colonnes étonnées». Il ne peut jamais passer indifférent devant les choses et les êtres de tous les jours. Les apparences quotidiennes des choses ne cessent d'être mystérieuses pour lui. Par delà elles il sait entendre des voix.

Supervielle est un poète du mystère inhérent à l'objet. Il ne fabrique pas d'ombre pour en envelopper sa vision ou sa pensée, afin de la rendre originale et rare; mais tout est pour lui imprégné d'invisible, et cet invisible il s'efforce de le rendre sensible, sous peine de défigurer la réalité totale. L'Univers n'est plus chez lui qu'un domaine affranchi de toute loi et qui attend le miracle où s'évanouissent toutes limites: entre le passé et l'avenir, le temps et l'espace, les choses et les hommes, le réel et le merveilleux, l'abstrait et le concret, le corps et l'âme, la vie et la mort...

Mais, quelle est, au juste, l'expérience que traduit la poésie sans frontières qu'il a conçue? En 1933, devant Jean Paulhan, il avoue:

«L'état de poésie me vient d'une sorte de confusion magique où les idées et les images se mettent à vivre, abandonnent leurs arêtes, soit pour faire des 
avances à d'autres images —dans ce domaine tout voisine, rien n'est vraiment éloigné-, soit pour subir de profondes métamorphoses, qui les rendent méconnaissables. Je me donne l'illusion de seconder l'obscur dans son effort vers la lumière, pendant qu'affleurent à la surface du papier les images qui bougeaient, reclamant dans les profondeurs» ${ }^{1}$.

Des images qui «se mettent à vivre», qui font «des avances à d'autres images», qui subissent de «profondes métamorphoses», dans una sorte de «confusion magique»: tout dans ce langage évoque l'«état de poésie», c'est-à-dire, l'inspiration poétique.

Le mot que Supervielle retient pour désigner cette «confusion magique» c'est le mot de RÊVE. Dès les premières lignes de son essai d'art poétique, il le met en valeur:

«La poésie vient chez moi d'un rêve toujours latent. (...) Rêver, c'est oublier la matérialité de son corps, confondre en quelque sorte le monde extérieur et l'intérieur. (...) Je rêve toujours un peu ce que je vois, même au moment précis et au fur et à mesure que je le vois» ${ }^{2}$.

Ces confidences impliquent une expérience fort étendue du rêve. Le poète qui la vit n'est pas seulement un homme qui se souvient de ses rêves nocturnes. Il continue de rêver au grand jour, les yeux ouverts. En effet, au lieu de parler de RÊVE, il faudrait parler plutôt de RÊVERIE.

Il est commun d'inscrire la rêverie parmi les phénomènes de la détente psychique. C'est une fuite hors du réel. En suivant «la pente de la rêverie», l'esprit se détend et se disperse et par conséquent s' «obscurcit». Mais un adjectif va tout sauver et nous permettre de dépasser les objections d'une psychologie primaire. Cette rêverie est la RÊVERIE POÉTIQUE. Une rêverie que la poésie met sur la bonne pente. C'est une rêverie qui s'écrit. Elle est confrontée au vertige de la page blanche. Alors les images se composent et s'ordonnent. Dans la rêverie poétique, tous les sens s'éveillent et s'harmonisent. Il y a donc un caractère constructif dans la rêverie poétique, qui n'est pas, en toute circonstance, un phénomène de détente et d'abandon comme le suggère la psychologie classique. C'est pourquoi il ne faut pas mettre, de façon arbitraire, la rêverie dans l'ordre des phénomènes oniriques. Il ne faut pas la considérer comme une simple dérivation du rêve. L'intervention possible de la conscience dans la rêverie apporte un signe décisif ${ }^{3}$.

Chez Supervielle, la rêverie poétique est une rêverie cosmique. Elle est une ouverture à un espace nouveau, le monde de la confiance. Cette rêverie naît de la solitude du poète à lui-même. Elle a sa racine dans l'âme du rêveur. Elle est, en définitive, un état d'âme.

Donc, Supervielle n'éprouve pas devant la solitude de révoltes désespérées. Il refuse l'infranchissable frontière qu'oppose au moi le non-moi. Il a le pressen-

1 Jean Paulhan, «Pour un tableau de la poésie en France», N.R.F., 1933, p. 704.

2 En songeant à un art poétique, Gallimard, 1951, pp. 57-58.

3 Voir à ce propos La poétique de la rêverie de Bachelard (P.U.F., 1960), dont la méthode phénoménologique permet de tirer au clair cette distinction entre le rêve et la rêverie. 
timent d'un monde «panthéiste. Le poète se trouve dans la certitude que le rêve est la révélation d'une réalité intérieure, semblable à toute réalité universelle, et que ses images tendent à lui restituer un monde plus proche du réel que celui des apparences. Supervielle cherche à établir dans ses poèmes cette communication avec le monde dont il est avide. Toute son oeuvre est une tentative de rapprochement universel. Il propose une grande famille cosmique où l'homme communique avec l'animal, le végétal et le minéral. Ses poèmes sont une tentative de vaincre l'espace et le temps, c'est-à-dire la solitude et l'isolement. Saisir le monde, le contenir, l'habiter, disparaître en lui..., en définitive un besoin obstiné de fusion; osmose à laquelle il parviendra, en détruisant les séparations qu'établissent la perception et la pensée. En écrivant, il dépasse la contradiction entre l'intérieur et l'extérieur, l'animé et l'inanimé, le visible et l'invisible. Dans cet état de liberté nouvelle, les cloisons entre le dehors et le dedans s'écroulent et la communion avec le monde redevient tout à fait possible.

Ainsi donc, Supervielle apporte une solution poétique au problème de la communication: il intègre le «monde» à son «moi» et de son «moi» il fait surgir une réalité vivante du «monde». La poésie est pour lui source d'existence. Elle est le seul moyen de vaincre l'universelle absence. Supervielle lui-même l'a dit:

«Et pourtant tout ce qui est interdit au poète dans la vie, lui devient possible et même recommandable dans une poésie transparente. (...) La poésie est pour les poètes l'art de ne se priver de rien» ${ }^{4}$.

Le monde réel a laissé donc place à un univers poétique existant par le langage, dans le langage. Supervielle récrée l'univers à sa guise, il défie l'impossible, il se rébelle contre toute loi limitant l'essor du désir et de la volonté de l'imaginaire.

Ce pouvoir magique de susciter le merveilleux où tout redevient libre, n'est autre que la fantaisie que Jules Supervielle définissait si heureusement dans une composition de son recueil intitulé Poèmes:

«Cueillir l'image où bon vous semble,

Happer des mots qui, d'abord, tremblent

De se trouver soudain ensemble,

Mais s'accoutument peu à peu...

Voler au gré de sa cervelle,

Changer la route d'un coup d'aile,

Avoir des tournants d'hirondelle!

Oublier qu'on n'a qu'une tête,

S'en croire mille, tant la fête

Est grande en l'âme du Poète!» ${ }^{5}$

Nous avons ici en germe, ce qui, à partir de Gravitations, deviendra le don de rebondir à travers le temps et l'espace. Pour le poète, siècles et distances s'abolissent, le surnaturel est apprivoisé, le domaine du possible agrandi. Les choses pensent, parlent, se déplacent; les êtres vivants n'obéissent plus à la loi de la pe-

4 «Lire des vers en public», Naissances, Gallimard, 1951, p. 70.

5 Poèmes, Paris, E. Figuière, 1919. 
santeur, traversent l'impénétrable, agissent par leur seule pensée, ont le don de survie, etc.

En effet, l'originalité la plus évidente de Supervielle est la vie de son imaginaire. Il a pris la décision de faire de l'insolite qui l'inquiétait, sa poésie, et son imagination apparaît comme une force et un mouvement de la conscience qui récrée le monde. Elle sonde les espaces maritimes et stellaires de même qu'elle pénètre au coeur des choses concrètes. Elle est capable d'animer l'inerte et de tout transfigurer à sa fantaisie. Au vrai rêveur rien d'impossible. Il se place au centre du monde, un monde dont il est à la fois le coeur et le démiurge.

Bachelard, dans son oeuvre L'air et les songes, nous dit:

«Pour la rêverie thaumaturge, tous les éléments sont bons, le monde entier peut s'animer sur l'ordre d'un regard magnétique. (...) Tout roule à souhait, des montagnes glissent, des avalanches s'écroulent, puis s'apaisent (...), tout l'univers se règle sur la volonté et l'imagination du rêveur» ${ }^{6}$.

Pour Supervielle, la poésie est la chance d'en finir avec la solitude, puisqu'elle établit entre les êtres une communication providentielle. Elle semble apporter une réponse définitive et heureuse aux angoisses et aux tristesses de la vie. Par l'écriture, le poète surmonterait la dispersion et la solitude, dans une promesse d'accomplissement. Le paradoxe du dire poétique est de naître de l'impossibilité même de communiquer.

Nous avons parlé de «rêverie», et qu'est-ce que c'est que rêver sinon la disposition à produire ou à accueillir des images? On a dit que rêver, c'est confondre en quelque sorte le monde extérieur et l'intérieur. Or, le lieu de cette «confusion» est l'IMAGE.

La réflexion même, chez lui, semble se produire par un surgissement de figures: «la plupart du temps, dit-il dans son Art Poétique, je n'avance dans ma pensée qu'à la faveur des images». Et un peu plus loin: «Si l'image, même quand elle est juste, est plus imprécise que le concept, elle rayonne davantage et va plus loin dans l'inconscient» ${ }^{7}$.

L'image joue donc un rôle privilégié dans le poésie de Supervielle. Elle est l'instrument de cette magie poétique. Dans les dernières pages du Corps tragique, nous trouvons encore ces quelques phrases avouant sa dette envers l'image:

«Il m'arrive souvent de me dire que le poète est celui qui cherche sa pensée et redoute de la trouver. La trouve-t-il qu'il pourrait bien cesser d'être un poète pour devenir un logicien, un prosateur, quelqu'un qui use d'abstractions pour s'exprimer. C'est dans une image, à l'avant-garde de lui-même, que le poète éprouve le besoin de fixer son esprit toujours en mouvement. Elle lui sert de relais jusqu'à ce que s'élève dans sa nuit personnelle une autre image qui se précise peu à peu. Ainsi se forme la chaîne de tout le poème» ${ }^{8}$.

La poésie est donc d'abord créatrice d'images et, grâce à la nature particulière de ces images, le poète a pu se composer un univers affranchi de tout détermi-

6 L'air et les songes, París, Corti, 1943, p. 213.

7 En songeant à un art poétique, p. 64.

8 Le corps tragique, Gallimard, 1959, p. 154. 
nisme, une fable à laquelle il impose ses lois propres. Dans cet univers où tout devient concret, palpable et visible, où rien ne veut demeurer, ni immobile, ni éloigné, les contraintes s'effacent. Le monde intérieur et le monde extérieur y échangent leurs signes et leurs attributs. L'âme du poète parcourt l'univers et celui-ci pénètre en elle. Il sent l'espace se prolonger en lui, avec tout ce qui le peuple:

«La campagne me devient presque tout de suite intérieure grâce à je ne sais quel glissement du dehors vers le dedans, à quoi ne participe pas seulement l'esprit, mais aussi les yeux, le nez, la bouche. Et j'ai l'impression d'avancer dans le paysage comme dans mon propre paysage mental» ${ }^{9}$.

L'ancienne distinction séparant le monde mental du monde extérieur est ainsi abolie: se prolongeant et se confondant, ils se livrent à d'étranges osmoses:

«Sa puissance circulaire rabat vers lui les lointains

Et l'on voit s'acheminer les étoiles scrupuleuses.

Le vieux sang noir de la nuit roule dans son propre sang

S'y mêlant au sang du jour dans l'abîme des cascades!

Tout s'absorbe et s'unifie dans son âme sans attente

L'univers n'est plus en lui qu'un grognement étouffé

Une famine allongée, ainsi qu'avant la Genèse» ${ }^{10}$.

Donc, Supervielle n'admet plus la distinction entre les objets de sa pensée et ceux du monde extérieur. Il en arrive à se concevoir comme un médiateur entre deux univers dont l'antagonisme n'est qu'une erreur de vision de la part de l'observateur. Sa logique le conduit à la certitude que le monde extérieur dépend entièrement de l'imagination qui le crée. L'espace où se meuvent les corps ne diffère en rien de celui où tournent désirs, souvenirs et sentiments. A l'omniprésence du poète correspond une présence en lui toujours possible de tout ce qui remplit l'univers.

Ainsi donc, il n'y a plus qu'un univers. Le monde extérieur impose trop sa présence pour rester complètement en dehors de la vie intérieure et entièrement séparé d'elle; et cette vie intérieure a trop d'effervescence et de richesse pour se maintenir dans ses limites et ne pas déborder.

Mais, sous cette allure fantastique, un souci du réel donne aux poèmes de Supervielle un humanisme très présent. Seuls de fréquents retours au réel et à l'humain rendent possibles les échappées dans le merveilleux. Supervielle aime qu'il y ait un sens à ce qu'il dit, qu'il y ait une logique, même poétique. Construit et animé par l'imagination, son univers n'est pourtant pas imaginaire. Bousculé, transformé, défiguré, il reste notre univers quotidien.

De même, ce que figurent les images les plus imprévues n'est jamais rien d'autre au fond que notre rapport véritable à ce réel. Elles ont parfois l'air de violer la réalité, mais ce qu'elles violent serait plutôt la vision de la réalité que notre vie ordinaire propose, le monde que notre intelligence nous habitue à percevoir. Ainsi, par exemple, dans son poème «47, Boulevard Lannes» il se trouve

9 Boire à la source, Gallimard, 1951, pp. 67-68.

10 «Aux creux du monde», Gravitations, Gallimard, 1978, p. 190. 
que l'appartement avec tout son mobilier est en plein ciel, ce qui n'est pas du tout habituel. Cependant, on observe, comme le fait remarquer Robert Vivier, «que non seulement tous les détails évoqués, les meubles par exemple, restent à la mesure de la perception quotidienne, mais que dans cette assomption d'apparence insolite, il n'y a en somme qu'une façon un peu catégorique de rendre évidente la situation physique très vraie qui est la nôtre à nous, emportés que nous sommes dans l'espace cosmique. Ce qui a donné naissance au poème (...) c'est une émotion de l'esprit devant le monde tel qu'il est» ${ }^{11}$.

Les images de Supervielle ne perdent jamais cette pesanteur sensible qui confère à sa poésie une si remarquable puissance de suggestion. Sa poétique reste liée à la démarche d'une rêverie irrépressible, certes, mais SURVEILLÉE. Comme le dit Octave Nadal, la pensée demeure «éveillée et pressentie, devinée sous la fiction» ${ }^{12}$.

L'image n'est jamais pour Supervielle un simple ornement décoratif. Comme le dit Yves-Alain Favre, Supervielle «sait que le poème est par essence inséparable de l'image. Mais, pour autant, il ne s'abandonne pas à l'enchantement des images et n'abdique pas devant leur pouvoir» ${ }^{13}$. Supervielle n'use que d'une image à la fois, en extrait le plus de signification possible et passe à une autre image; mais il veut que ce passage possède une cohérence et une plausibilité, et que le poème soit une architecture d'images. Supervielle lui-même nous dit:

«Il n'y a pas que les images. Il y a les passages des unes aux autres qui doivent être aussi de la poésie. (...) Je compte sur mon poème pour ordonner et faire chanter juste les images» ${ }^{14}$.

Supervielle a toujours eu le souci de maîtriser une matière insolite dans une langue qui fût comprise par tous. Il fuit l'image pour l'image. Il la soumet au contrôle de la conscience, si bien que le rôle de l'image chez lui serait de servir de synthèse dans la dialectique rêve / raison. L'image de Supervielle n'a jamais pour but de décorer ou de renforcer l'expressivité, mais, d'éclairer l'imprécis et d'illuminer l'obscur. Elle est, d'après les propres mots du poète, «la lanterne magique qui éclaire les poètes dans l'obscurité» ${ }^{15}$. Loin d'être un jeu superficiel, ce langage naturel et lumineux l'aide non seulement à tirer au clair son propre chaos intérieur, mais encore à se faire comprendre de tous ses lecteurs.

Naturellement obscur, Supervielle s'est toujours efforcé d'aller vers la clarté. C'est sans doute par cette raison qu'il a écrit pour le théâtre, car il faut que la pièce soit immédiatement communicable. De même, c'est peut-être cette impulsion réaliste qui a produit la tendance de la poésie superviellienne à la fabulation, ou, en tout cas, a donné à celle-ci les moyens de se manifester. Et il ne serait pas invraisemblable d'y rattacher le fait que tant de poèmes déploient une intuition narrative, soient des ébauches de récit.

11 Robert Vivier, Lire Supervielle, Corti, 1971, pp. 23-24.

12 «Conversation avec Supervielle», N.R.F., oct. 1960, p. 630.

13 La rêverie et le chant dans Gravitations, Paris, Nizet, 1981, p. 47.

14 En songeant à un art poétique, p. 61.

15 Ibídem. 
En effet, le conte a beaucoup aidé sa poésie, parce qu'il doit concilier beaucoup mieux les exigences de la fantaisie et celles de la raison. Comme le dit Bachelard, «l'imagination qui raconte doit penser à tout. Elle doit être plaisante et sérieuse, elle doit être rationnelle et rêveuse. (...) Le conte le meilleur est celui qui sait toucher les limites de la crédulité» ${ }^{16}$. Le conteur surveille donc le poète. En effet, beaucoup de poèmes commencent comme des contes. Le récit n'est là que pour soutenir les démarches de la rêverie. Son rôle est de rassurer le lecteur, de lui donner «l'illusion de la vérité».

On a beaucoup parlé de fantastique chez Supervielle, et aussi de fantaisie, d'hallucination, etc. Nous ne sommes pas d'accord. Certes, son imagination est prolixe, mais son terrain n'est pas le fantastique ni l'hallucination, mais celui d'une appréhension du réel au moyen d'images qui, sous l'apparence d'irréalisme, rendent compte d'une interrogation des plus sérieuses portant sur ce réel. L'imagination ne quitte donc pas la main de l'observation. Supervielle ne s'évade pas dans des mondes de fantaisie ou de pure hallucination: ce qui l'hallucine c'est la réalité. Pour cela, Claude Roy affirme: «Les poèmes de Supervielle constituent un univers d'analogies, mais non pas un univers fabuleux» ${ }^{17}$.

Tout paré qu'il soit des prestiges de la fantisie et de l'imagination, le monde de Supervielle est un monde de la présence, un monde éprouvé autant qu'imaginé; si bien que les rêveries les plus insolites nous paraissent finalement naturelles et vraisemblables.

La conscience demeure toujours vigilante. «Pour moi, le poète, dit Supervielle, doit être éveillé tout en gardant des lambeaux de sommeil conscient, je veux dire de sommeil rêveur et imagé» ${ }^{18}$. Il perçoit la menace du délire. Il ne s'y abandonne jamais. Il faut dégager du chaos des rêves la fable qui les gouverne en secret. Supervielle a besoin, à la différence d'autres auteurs (on pense aux surréalistes), du secours de l'intelligence:

«Je n'aime pas le rêve qui s'en va à la dérive, j'allais dire: à la dérêve. Je cherche à en faire un rêve consistant, une sorte de figure de proue qui, après avoir traversé les espaces et les temps intérieurs, affronte les espaces et les temps du dehors —et pour lui, le dehors c'est la page blanche» ${ }^{19}$.

Supervielle tend à ce que le surnaturel devienne naturel. Il agit en sorte que l'ineffable nous devienne familier tout en gardant ses racines fabuleuses. D'où la boutade qu'il lança un jour, excédé par les extravagances du surréalisme:

«André Breton déclare que tout critique qui n'admet pas qu'un cheval puisse galoper dans une tomate est imbécile. Eh bien! ce qui m'intéresse, c'est de savoir comment le cheval est entré dans la tomate, c'est le passage de l'humain au divin. Ce qui m'intéresse c'est le moment où ça décolle» ${ }^{20}$

16 La terre et les rêveries du repos, Corti, 1948, p. 129.

17 Jules Supervielle, Seghers, 1949, p. 22.

18 O. Nadal, «Conversation avec Supervielle», op. cit., pp. 625-26.

19 En sorgeant à un art poétique, p. 57.

20 Cité sans référence par Paul Viallaneix dans son livre Le Hors-venu, Klincksieck, 1972, p. 41. 
Supervielle a toujours aspiré à la cohérence et à la plausibilité, à ne jamais rompre le lien avec la réalité, à conserver un langage continu, un agencement plausible. Sa poésie est née du rêve, certes, mais aussi de la peur du rêve. Il a toujours redouté les forces de dislocation et de démence qui guettent le poète qui se laisse aller. Bref, sa poésie se situe à la rencontre des élans vagues et des exigences lucides. C'est la poésie d'un rêveur qui reste attentif. 